\title{
Analytical Representation of Total Cross-Sections for Electron Atom Scattering: Application to Atomic Helium
}

\author{
A. Mansouri ${ }^{a}$, S. Hounmer ${ }^{a}$ And M. MoulaY ${ }^{b}$ \\ ${ }^{a}$ Département de physique — Faculté des sciences \\ Université Ferhat Abbas, Sétif, Algeria \\ ${ }^{b}$ CRNA 2, Bd Frantz Fanon, B.P. 399, Alger-gare, Algeria
}

(Received July 30, 2002; revised version April 29, 2003)

\begin{abstract}
Based on the series expansion formalism, an analytical approach is proposed to evaluate the total cross-sections induced by electron impact excitation. As an illustration, an analytical expression of the total cross-section of the double excitation of helium atom by electron impact is obtained for the $2 p^{2}{ }^{3} P$ and $2 p 3 p{ }^{1} P$ transitions within the framework of the distorted wave Born approximation. The available experimental data are well reproduced by the obtained expression of the total cross-section which is function of the only parameter of the incident electron energy. Comparisons are also made with numerical calculations.
\end{abstract}

PACS numbers: 31.15.-p, 02.70.-c

\section{Introduction}

In many applications the cross-sections are used as input data in the form of tables of sparsely distributed values or given in a limited range [1]. In electron-atom collisions phenomena, these quantities play a central role for the understanding of the physical structure of atoms. Several methods have been proposed to treat this process numerically over the past three decades [2-5]. When the compared results are in agreement with the data then the used methods are considered as powerful and reliable. However, when the data are not satisfactorily described, one usually tries to include corrections or to improve the employed numerical methods before 
introducing different corrections to improve the description of the cross-sections. There are many sources of uncertainties in the evaluation of scattering amplitude. Among them, the approximate method adopted for calculation, the inaccurate input wave functions describing the target, the treatment of the radial wave equations, and the singularity problems.

In this paper we propose an analytical approach for evaluating the differential cross-section without resorting to extensive numerical approximate calculations. Our purpose is to obtain an analytical representation of the $T$-matrix element describing the excitation of atoms from the ground state. The aim is, on the one hand, to avoid the uncertainties related to the use of the numerical methods and divergence problems and, on the other hand, to obtain an analytical representation of the cross-sections which may be required in analytical form in more complicated calculations [1]. To illustrate the proposed approach, we consider the $2 p^{2}{ }^{3} P$ and $2 p 3 p{ }^{1} P$ transitions corresponding to the electron-impact double excitation of the helium atom from its ground state.

This paper is organized as follows. In Sec. 2 an analytical solution of the Schrödinger equation is constructed using the Frobenius series expansion. The analytical representation of the cross-section is obtained in Sec. 3. The results are presented and discussed in Sec. 4.

\section{Theoretical framework}

The $T$-matrix element for the electron impact excitation of an $N$-electron target atom is given (in atomic units) by [6]:

$$
\begin{aligned}
& T_{\mathrm{if}}=-\frac{1}{2 \pi} \\
& \times\left\langle\chi_{\mathrm{f}}^{-}(0) \Psi_{\mathrm{f}}(1,2, \ldots, N)\left|V(1,2, \ldots, N)-U_{\mathrm{f}}(0)\right| A \Psi(1,2, \ldots, N) \chi_{\mathrm{i}}^{+}(0)\right\rangle,
\end{aligned}
$$

where $\chi_{\mathrm{i}}^{+}(0)$ and $\chi_{\mathrm{f}}^{-}(0)$ are the distorted wave functions of the electron in the incident and exit channels, respectively, and $0,1,2, \ldots, N$ refer to position vectors $r_{0}, r_{1}, \ldots, r_{N}$ :

$$
\chi^{ \pm}(\boldsymbol{r})=\sqrt{\frac{2}{\pi}} \frac{1}{k r} \sum_{l, m}( \pm \mathrm{i})^{l} \frac{2 l+1}{4 \pi} \chi_{l}(k, r) P_{l}(k, r),
$$

$\Psi_{\mathrm{i}}$ and $\Psi_{\mathrm{f}}$ are the antisymmetrized initial and final atomic wave functions, respectively, and $V$ is interaction potential between the projectile electron and the target atom with a charge $Z$ :

$$
V=-\frac{Z}{r_{0}}+\sum_{i=1}^{N} \frac{1}{r_{i 0}}
$$

where $r_{i 0}$ is the distance between the bound electron $i$ and the incident electron located at $r_{0}$. The antisymmetrization operator $A$ : 


$$
A=\frac{1}{N+1}\left(1-\sum_{i=1}^{N} P_{i 0}\right)
$$

where $P_{i 0}$ is the exchanges electron operator. Neglecting the exchange and polarization effects, the distorting potential $U_{\mathrm{i}(\mathrm{f})}$ in the incident (exit) channel can be written as $U_{\mathrm{i}(\mathrm{f})}=V_{\mathrm{i}(\mathrm{f})}^{\text {stat }}$ where

$$
V_{\mathrm{i}(\mathrm{f})}^{\mathrm{stat}}(r)=-\frac{Z}{r}+\sum_{n, l} N_{n, l} \int \frac{R_{n l}\left(r^{\prime}\right)}{\max \left(r, r^{\prime}\right)} r^{\prime 2} \mathrm{~d} r^{\prime}
$$

is the standard Hartree potential. $N_{n j}$ is the number of electrons in the considered orbital and $R_{n l}(r)$ - the radial atomic wave function. The radial distorted waves $\chi_{l}(k, r)$ are solution of the Schrödinger equation

$$
\left[\frac{\mathrm{d}^{2}}{\mathrm{~d} r^{2}}-\frac{l(l+1)}{r^{2}}-2 U_{\mathrm{i}(\mathrm{f})}(r)+k_{\mathrm{i}(\mathrm{f})}^{2}\right] \chi_{l}\left(k_{\mathrm{i}(\mathrm{f})}, r\right)=0
$$

with the boundary conditions

$$
\chi_{l}(k, 0)=0 \quad \text { and } \quad \chi_{l}(k, r)_{r \rightarrow \infty}=\frac{1}{\sqrt{k}} \sin \left(k r-\frac{l \pi}{2}+\delta_{l}\right),
$$

$\delta_{l}$ being the phase shift of the $l$-th partial waves. The differential cross-section for the inelastic process can be written as

$$
\frac{\mathrm{d} \sigma}{\mathrm{d} \Omega}=\frac{k_{\mathrm{f}}}{k_{\mathrm{i}}}\left|T_{\mathrm{if}}\right|^{2}
$$

The $T_{\text {if }}$ matrix elements are derived from (1). The total cross-section $\sigma$ can be obtained by averaging over initial spin states, summing over final spin states and integrating over all scattering angles. Our main task is to derive an analytical form of the $T_{\text {if }}$ matrix elements given in Eqs. (1) and (7). At the first stage, an analytical form of the radial distorted waves $\chi_{l}(k, r)$ in $(5)$ can be obtained using Frobenius series expansion. The latter have been successfully applied to solve the eigenvalue problem for the Coulombic model potential [7].

Let us consider the following differential homogeneous equation:

$$
y^{\prime \prime}(x)+\frac{P(x)}{x-x_{0}} y^{\prime}(x)+\frac{Q(x)}{\left(x-x_{0}\right)^{2}} y(x)=0
$$

with a regular singular point at $x=x_{0} . P(x)$ and $Q(x)$ can be expanded as the Taylor series about $x_{0}$ :

$$
P(x)=\sum_{n=0}^{\infty} p_{n}\left(x-x_{0}\right)^{n}, \quad Q(x)=\sum_{n=0}^{\infty} q_{n}\left(x-x_{0}\right)^{n} .
$$

Hence, a solution of the differential equation (8) can be written as a Frobenius series

$$
y_{1}(x)=\sum_{n=0}^{\infty} a_{n}\left(x-x_{0}\right)^{n+\alpha}
$$


where $\alpha$ is an indicial exponent to be determined from the following relations:

$$
\begin{aligned}
& P_{0}(\alpha) a_{n}=0, \\
& P_{n}(\alpha) a_{n}+\sum_{k=0}^{n-1}\left[(\alpha+k) p_{n-k}+q_{n-k}\right] a_{k}=0, \\
& P_{n}(\alpha)=(\alpha+n)^{2}+\left(p_{0}-1\right) \alpha+q_{0} .
\end{aligned}
$$

Since $a_{0} \neq 0$, it follows that:

$$
P_{0}(\alpha)=\alpha^{2}+\left(p_{0}-1\right) \alpha+q_{0}=0 .
$$

If $\alpha_{1}$ and $\alpha_{2}$ are the roots of $P_{0}(\alpha)$ with $\alpha_{1}-\alpha_{2}=N$, where $N$ is an integer, then Eq. (8) has a second linearly independent solution

$$
y_{2}(x)=\sum_{n=0}^{\infty} c_{n}\left(x-x_{0}\right)^{\alpha_{2}+n}-\left[a_{n}^{\prime}+a_{n} \ln (x)\right]\left(x-x_{0}\right)^{\alpha_{1}+n}
$$

with the following recurrence relations:

$$
\begin{aligned}
& a_{n}^{\prime}\left(\alpha_{1}\right)=\left.\frac{\partial a_{n}}{\partial \alpha}\right|_{\alpha=\alpha_{1}}, \\
& P_{n}\left(\alpha_{1}\right) a_{n}+\sum_{k=0}^{n-1}\left[\left(\alpha_{1}+k\right) p_{n-k}+q_{n-k}\right] a_{k}=0, \quad n \neq 0, \\
& P_{n}\left(\alpha_{21}\right) c_{n}+\sum_{k=0}^{n-1}\left[\left(\alpha_{2}+k\right) p_{n-k}+q_{n-k}\right] c_{k}=0, \quad n \neq 0, N, \\
& \sum_{k=0}^{N-1}\left[\left(\alpha_{2}+k\right) p_{N-k}+q_{N-k}\right] c_{k}=P_{0}^{\prime}\left(\alpha_{1}\right) a_{0}\left(\alpha_{1}\right),
\end{aligned}
$$

$c_{0}$ and $c_{N}$ are defined by the boundary conditions and $a_{0}$ is determined by relation

(14d). Finally, the analytical solution of (8) is

$$
y(x)=\sum_{n=0}^{\infty}\left\{c_{n}\left(x-x_{0}\right)^{\alpha_{2}+n}-\left[a_{n}^{\prime}+a_{n} \ln (x)+a_{n}\right]\left(x-x_{0}\right)^{\alpha_{1}+n}\right\} .
$$

Applying the above procedure to Eq. (5), we can write

$$
\chi_{l}^{\prime \prime}(k, r)+\frac{Q(r)}{r} \chi_{l}(k, r)=0 .
$$

Comparing with Eq. (8) we have

$$
P(r)=0, \quad Q(r)=\sum_{n=0}^{\infty} g_{n} r^{n} \quad \text { with } \quad q_{n}=\frac{1}{n !} \frac{\mathrm{d}^{\prime \prime} Q}{\mathrm{~d} r^{n}} .
$$

The indicial equation (12) gives

$$
P_{0}(\alpha)=\alpha^{2}-\alpha-l(l+1)=0,
$$

yielding

$$
\alpha_{1}=l+1, \quad \alpha_{2}=-l .
$$


In our case, $\alpha_{1}-\alpha_{2}=2 l+1$ is an integer. The appropriate corresponding distorted wave function $\chi_{l}(k, r)$ is of the form (15). The coefficients $a_{0}, c_{0}, a_{n}, a_{n}^{\prime}, c_{n}$ and $c_{N}$ are evaluated using the recurrence relations (14) and taking into account (6).

\section{The analytical representation of the cross-section for the $2 p^{2}{ }^{3} P$ and $2 p 3 p^{1} P$ transitions of helium atom}

The method developed above is applied to evaluate the double excitation cross-section of helium. The parity unfavored longer lived states [8, 9] states $2 p^{2}{ }^{3} P$ and $2 p 3 p{ }^{1} P$ are considered. An analytical form of the matrix element given by Eq. (1) can be derived using symmetrized hydrogenic wave functions $\Psi_{n l m}(\boldsymbol{r})$ and taking into account relations (2), (3) and (4). The distorted wave function $\chi_{l}(k, r)$, solution of Eq. (5), is taken in the form of the Frobenius series given by (15) under the boundary conditions (6). By comparing with the corresponding numerical solution of (15), it turns out that the analytical solution is sufficiently accurate for the first four terms in the series (15). In Table, several values of the numerical solution of Eq. (5) and the analytical one with respectively two, three, and four terms, are displayed for purpose of comparison. We can see that analytical solutions with two and three terms are not convenient, while

\section{TABLE}

Values of numerical and analytical solution of differential equation (5), respectively noted $u(r)$ and $p u(r)$ with 2,3 , and 4 terms for incident electron energy $E_{\mathrm{i}}=60 \mathrm{eV}$, with respect to the position $r$.

\begin{tabular}{l|c|c|c|c}
\hline \hline & $r[\AA]$ & $\begin{array}{c}\text { Numerical } \\
\text { solution } u(r)\end{array}$ & $\begin{array}{c}\text { Analytical } \\
\text { solution } p u(r)\end{array}$ & Abs $[u(r)-p u(r)]$ \\
\hline 2 terms & 0.5008 & 0.00866 & -0.01072 & 0.01938 \\
& 0.8339 & 0.11800 & -0.02985 & 0.14790 \\
& 1.1670 & 0.51330 & 0.04526 & 0.46800 \\
& 1.5000 & 1.11300 & 0.47370 & 0.63950 \\
& 1.8330 & 1.294 & 1.70400 & 0.40980 \\
\hline \multirow{3}{*}{ terms } & 0.5008 & 0.00866 & -0.08741 & 0.09607 \\
& 0.8339 & 0.11800 & -0.01725 & 0.13530 \\
& 1.1670 & 0.51330 & 0.49200 & 0.02125 \\
& 1.5000 & 1.11300 & 1.29600 & 0.18280 \\
& 1.8330 & 1.29400 & 1.06000 & 0.23360 \\
\hline & 0.5008 & 0.00866 & 0.01171 & 0.00305 \\
& 0.8339 & 0.11800 & 0.12090 & 0.00286 \\
& 1.1670 & 0.51330 & 0.48990 & 0.02339 \\
& 1.5000 & 1.11300 & 1.13700 & 0.02411 \\
& 1.8330 & 1.29400 & 1.28000 & 0.01383
\end{tabular}


with four terms the agreement is good. Frobenius expansions are called convergent series, obviously, the results are more accurate by using more terms in the expansion. In the studied transitions results were sufficiently convergent with at least four terms. Hence, the expansion has been truncated to the fourth term just to illustrate the proposed method with a minimum number of terms. Formula (14) enables to deduce other terms to be used in the expansion if more accuracy is required. The phase shifts $\delta_{l}$ appearing in conditions (6), are calculated using the well known results of scattering quantum theory in the first-order approximation and including the asymptotic region solution. The interaction potential is of short range in the considered problem. The asymptotic region is then reached even for short distances. Frobenius solutions are used around zero, i.e., at short distances and the use of phase shifts can then be justified in this case. Once the functions $\chi_{l}(k, r)$ are completely defined in the form of a Frobenius series, the analytic expression of the matrix elements $T_{\text {if }}$ and then the differential cross-section defined by relation (7) are obtained performing spatial integration and taking the initial direction of the free electron as reference for the scattering angle. The derived analytical function depends on the unique parameter which is the electron impact energy.

The $1 s^{2}{ }^{1} S \rightarrow 2 p^{2}{ }^{3} P$ transition is described by the following relations:

$$
\begin{aligned}
& \Psi_{\mathrm{i}}(1,2)=\Psi_{100}\left(\boldsymbol{r}_{1}\right) \Psi_{100}\left(\boldsymbol{r}_{2}\right), \\
& \Psi_{\mathrm{f}}(1,2)=\frac{1}{2} \sum_{m_{1}, m_{2}}\left\langle 1 m_{1} 1 m_{2} \mid 1, M\right\rangle \Psi_{21 m_{1}}\left(\boldsymbol{r}_{1}\right) \Psi_{21 m_{2}}\left(\boldsymbol{r}_{2}\right), \\
& \sigma=3 \frac{k_{\mathrm{f}}}{k_{\mathrm{i}}} \int\left|T_{\mathrm{if}}\right|^{2} \mathrm{~d} \Omega .
\end{aligned}
$$

Integrating over scattering angle $\theta=\left(\boldsymbol{k}_{\mathrm{i}}, \boldsymbol{k}_{\mathrm{f}}\right)$ gives

$$
\sigma=\frac{1}{k_{\mathrm{i}}^{3} k_{\mathrm{f}}}\left(I_{1} I_{2}\right)^{2},
$$

where $I_{1}$ and $I_{2}$ are defined by

$$
\begin{aligned}
& I_{1}=\sum_{n=0}^{4} \alpha_{n+1} c_{n}-\sum_{n=0}^{4} \alpha_{n+4} a_{n}^{\prime}-\sum_{n=0}^{4} \beta_{n+4} a_{n} \\
& I_{2}=\sum_{n=0}^{4}\left(24 \alpha_{n-2}-\varepsilon_{n}\right) c_{n}-\sum_{n=0}^{4}\left(24 \alpha_{n+1}-\varepsilon_{n+3}\right) a_{n}^{\prime}-\sum_{n=0}^{4}\left(24 \beta_{n+1}-\delta_{n}\right) a_{n} .
\end{aligned}
$$

Here, $\alpha_{n}, \beta_{n}$ and $\omega_{n}$ are given by

$$
\begin{aligned}
& \alpha_{n}=\Gamma(n+1), \quad \beta_{n}=\Gamma(n+1) \gamma(0, n+1), \\
& \varepsilon_{n}=\sum_{i=-1}^{2} \nu_{i+1}\left(\frac{2}{5}\right)^{n+i-1} \Gamma(n+i-1), \quad n \geq 2, \\
& \varepsilon_{0}=380.41, \quad \varepsilon_{1}=50.81,
\end{aligned}
$$




$$
\delta_{n}=\sum_{i=1}^{4} \nu_{4-i}\left(\frac{2}{5}\right)^{n+i+1} \Gamma(n+i+1)\left[-\ln \left(\frac{2}{5}\right)+\gamma(0, n+i+1)\right],
$$

where $\gamma(n, z)$ is the polygamma function defined by $\gamma(n, z)=\mathrm{d}^{n} \Psi(z) / \mathrm{d} z^{n}$ with $\Psi(z)=\Gamma^{\prime}(z) / \Gamma(z) ; \Gamma(z)$ being the usual gamma function. In our case, the coefficients $\nu_{i}$ are given by

$$
\nu_{0}=10.125, \quad \nu_{1}=27, \quad \nu_{2}=36, \quad \nu_{3}=24, \quad \nu_{4}=0 .
$$

In the case of the $1 s^{2}{ }^{1} S \rightarrow 2 p 3 p{ }^{1} P$ transition, we have

$$
\begin{aligned}
& \Psi_{\mathrm{i}}(1,2)=\Psi_{100}\left(r_{1}\right) \Psi_{100}\left(r_{2}\right) \\
& \Psi_{\mathrm{f}}(1,2)=\frac{1}{\sqrt{2}} \sum_{m_{1}, m_{2}}\left\langle 1 m_{1} 1 m_{2} \mid 1 M\right\rangle \\
& \quad \times\left[\Psi_{21 m_{1}}\left(\boldsymbol{r}_{1}\right) \Psi_{31 m_{2}}\left(r_{2}\right)+\Psi_{21 m_{1}}\left(r_{1}\right) \Psi_{31 m_{2}}\left(r_{2}\right)\right] .
\end{aligned}
$$

The total cross-section obtained is

$$
\sigma=\frac{k_{\mathrm{f}}}{k_{\mathrm{i}}} \int\left|T_{\mathrm{if}}\right|^{2} \mathrm{~d} \Omega=\frac{1}{k_{\mathrm{i}}^{3} k_{\mathrm{f}}}\left(I_{1} I_{2}-J_{1} J_{2}\right)^{2},
$$

where

$$
\begin{aligned}
I_{1} & =\sum_{n=0}^{4} \alpha_{n+1} c_{n}-\sum_{n=0}^{4} \alpha_{n+4} a_{n}^{\prime}-\sum_{n=0}^{4} \beta_{n+4} a_{n}, \\
I_{2} & =\sum_{n=0}^{4}\left(72 \alpha_{n-2}+\lambda_{n}\right) c_{n}-\sum_{n=0}^{4}\left(72 \alpha_{n+1}+\lambda_{n+3}\right) a_{n}^{\prime}-\sum_{n=0}^{4}\left(72 \beta_{n+1}+\chi\right) a_{n}, \\
J_{1} & =\sum_{n=0}^{4} \rho_{n} c_{n}-\sum_{n=0}^{4} \rho_{n+3} a_{n}^{\prime}-\sum_{n=0}^{4} \mu_{n+4} a_{n}, \\
J_{2} & =\sum_{n=0}^{4}\left(24 \alpha_{n-2}-\varepsilon_{n}\right) c_{n}-\sum_{n=0}^{4}\left(24 \alpha_{n+3}-\varepsilon_{n+3}\right) a_{n}^{\prime}-\sum_{n=0}^{4}\left(24 \beta_{n+1}-\delta_{n}\right) a_{n}
\end{aligned}
$$

with

$$
\begin{aligned}
\rho_{n} & =-\frac{n}{2} \Gamma(n+2), \quad \mu_{n}=\frac{\Gamma(n+1)}{2}[(1-n) \gamma(0, n+1)-1], \\
\lambda_{n} & =\sum_{i=-2}^{2} w_{2+i}\left(\frac{2}{7}\right)^{n+1+i} \Gamma(n+i+1) \\
& \times\left[-\ln \left(\frac{7}{2}\right)+\gamma(0, n+i+1)\right], \quad n \geq 2,
\end{aligned}
$$




$$
\begin{aligned}
& \lambda_{0}=306.32, \quad \lambda_{1}=-177.76 \\
& \chi_{n}=\sum_{i=1}^{5} w_{i-1}\left(\frac{2}{5}\right)^{n+1+i} \Gamma(n+1+i)\left[-\ln \left(\frac{5}{2}\right)+\gamma(0, n+1+i)\right] .
\end{aligned}
$$

In this case the coefficients $w_{i}$ are given by

$$
w_{0}=72, \quad w_{1}=-96, \quad w_{2}=-64, \quad w_{3}=28.44, \quad w_{4}=9.48 .
$$

\section{Results and discussions}

Analytical representation of the total cross-section have been obtained for $2 p^{2}{ }^{3} P$ and $2 p 3 p{ }^{1} P$ excitations of helium without resorting to numerical approximate calculations or introducing adjustable parameters. In Fig. 1, the calculated total cross-section for the $2 p^{2}{ }^{3} P$ excitation from the ground state is displayed from threshold up to $140 \mathrm{eV}$. The results obtained by using Eq. (23) are compared with experimental data [10] and with the results given by the distorted wave polarized orbital (DWPO) model [11]. It is seen that the present results are in good agreement with the data. The near-threshold behavior and the maximum of the total cross-section as a function of impact electron energy are well reproduced. Near the threshold region the DWPO results are somewhat shifted. However, the position of the respective maxima are about the same in magnitude. As expected, at higher energies all the results approach the same asymptotic limit.

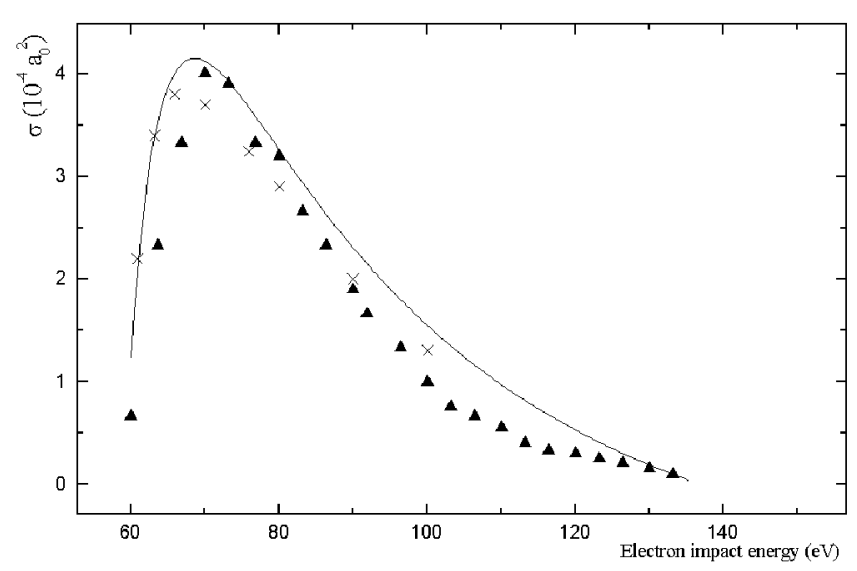

Fig. 1. Total cross-section for $2 p^{2}{ }^{3} P$ excitation of He atom by electron impact. Solid curve - present results, cross points - experimental results [10], up triangles points DWPO results [11]. 


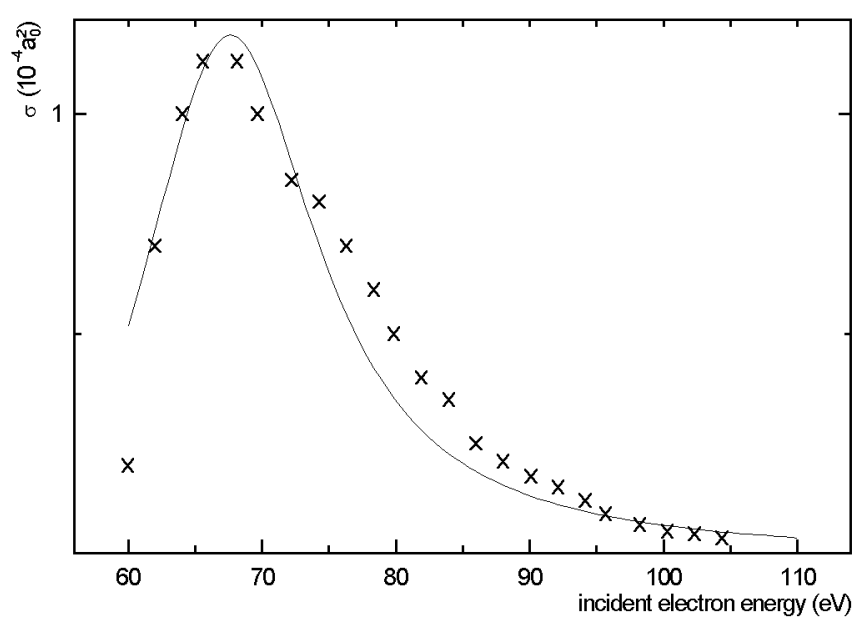

Fig. 2. Total cross-section for $2 p 3 p{ }^{1} P$ excitation of He by electron impact. Solid curve - present results, cross points - BO theory results [12].

Figure 2 shows the total cross-section obtained in the proposed approach for the $2 p 3 p{ }^{1} P$ excitation of He-atom with an incident electron energy ranging from threshold up to $110 \mathrm{eV}$ (asymptotic region). Our results are compared with those of the Born-Oppenheimer (BO) theory [12] with hydrogenic orbital wave functions. It is seen that results obtained from Eq. (26) and those of BO theory are very close to each other. The peak values are about the same in magnitude and location and the behavior is similar for both approaches in almost the entire energy region. To our knowledge there is no data for this transition.

Comparing all results among themselves, it appears that an analytical treatment of such problems can be carried out before any definite conclusion regarding the different corrections than can be made to improve the description of the cross-sections. The proposed approach allows to diminish the discrepancies related to the sources of uncertainties. As mentioned above, the analytical solution of the total cross-section has been obtained using four terms in the series (15) representing the radial distorted wave. Obviously, high accuracy results can be obtained by using more terms of the considered expansion.

In conclusion, we have shown that the total cross-section of the electron impact double excitation can be obtained analytically in the framework of the distorted wave approximation. Two specific transitions of helium were considered. The used procedure based on the Frobenius series expansion, can be extended to other similar excitations of atoms. The analytical results obtained for the total cross-sections depend on the unique parameter, that is the electron impact energy. 


\section{Acknowledgment}

We are very grateful to Dr H. Mekis for fruitful discussions.

\section{References}

[1] M. Inokuti, M. Kimura, M.A. Dillon, I. Shimamura, Adv. At. Mol. Opt. Phys. 33, 215 (1994).

[2] S. Chung, C.C. Lin, E.T.P. Lee, Phys. Rev. A 12, 1340 (1975).

[3] K.A. Berrington, P.G. Burke, A.L. Sinfailam, J. Phys. B 8, 1459 (1975).

[4] M.T. Lee, L.E. Machado, L.M. Brescansen, G.D. Meneses, J. Phys. B 24, 509 (1991).

[5] D.V. Fursa, I. Bray, J. Phys. B 30, 5895 (1997).

[6] K. Bartschat, Computational Atomic Physics, Springer-Verlag, Berlin 1996, p. 65.

[7] S.D. Chao, S.H. Lin, Eur. Phys. J. D 7, 475 (1999).

[8] K.C. Kulander, J. Dahler, Phys. Rev. A 6, 1436 (1972).

[9] D.L. Hickerson, L. Mlodinov, J.S. Dahler, J. Phys. B 11, 2377 (1978).

[10] W.B. Westerveld, F.B. Kets, H.G.M. Heideman, J. Van Eck, J. Phys, B 12, 2575 (1979).

[11] R. Srivastava, M. Kumar, Phys. Rev. A 31, 3639 (1985).

[12] M. Becker, J.S. Dahler, Phys. Rev. A 73, 136 (1964). 